\title{
Nanone Interactions in Antibody of Living Systems
}

\author{
Rajasekaran Ekambaram ${ }^{1}$ \\ ${ }^{1}$ Department of Chemistry, V.S.B. Engineering College, India \\ Correspondence: Rajasekaran Ekambaram, Department of Chemistry, V.S.B. Engineering College, Karur - \\ 639111, TN, India. E-mail: ersekaran@gmail.com
}

Received: November 8, 2021; Accepted: December 7, 2021; Published: December 16, 2021

\begin{abstract}
One of the facts about how nanoparticle assemble and act is revealed using carbon value in biomolecule of living system here. This is how the biomolecules interact to bring about a micro or even macro level interaction in system of interest. This study shows micro level understanding can be better utilized from carbon analysis at nano level. I plan to extend this phenomena of change from nano to micro for building large scale applications in human nature. Applications include corrections in both at sequence and structure level for permanent recovery of defective one, adding flavor to the existing biomolecule for faster delivery or recovery etc. I have demonstrated here the active role played by carbon and all. This might be extended to another system of setup where new applications yet to be created. One can extend this phenomena of change from nano to large scale one.
\end{abstract}

Keywords: internal COD, cohesive force, nanoparticle, nanone interaction, immunoglobulin

\section{Introduction}

One of the force coming from carbon is revealed in recent literature [1-8] that all interaction living being made up of carbon force out of the context of intervening molecules coexistence together with other force of interaction. Interestingly the idea of intervening molecules in the vicinity of interaction is seems to be the interactions coming from carbon alone and all other forces of interactions are negligibly small quantity. At this juncture it is believed to be the nano level force coming out of carbon value is interestingly important in interpretation of existing interactome in the living being. Existence of this force arising from carbon adequately explains the phenomena of interaction $[6,7]$ in the vicinity of interaction as explainable from protein-probe interaction [8] during biochemical reactions of interacting biomolecules at the juncture of invisible sites of interaction. In this context it is necessary to examine the role played by antigen antibody interaction to study the biological function of disease recovery. Analyzing this fact of carbon role in this study on antigen antibody interaction is crucial for other alteration that can take place for mutational one in the years to come. Authentication has to be carried out at the level of carbon point of view rather than any other force coming into existence during interaction in biomolecular systems of setup for biological function in living being. Additionally it is important to note that equal amount of intervening force out of other system needs to be taken care otherwise it is negligence of overall performance of intervening molecules during evolution and all. Otherwise it is a mere force of interaction coming from carbon alone and all. Over and above it is important to note that other kind of dealing this force of attraction result in jeopardize the system during calculations. Once it is noted from other fabrication of attraction is used, it is clear evidence that it may not survive further with other kind of interaction during fragmentation and analysis.

Working principle of carbon value [9-13] to intervening molecule of interaction during antigen antibody interaction is the focus of this work here. Very much into force of attraction in the vicinity of active line is focused. That is immunoglobulin light chain variable is taken here as sample work and studied all relevant role of carbon here in interaction. Existing role played by carbon in this intervening molecule is studied thoroughly and all revelation are put forth for further analysis or improvement over alteration principle begins to start.

The $\mathrm{FAB}$ one that is fragment antigen binding region that and all including variable and constant parts are taken here for further processing the disease solving problem of interactive domain one [14-16]. The variable one is taken here for including in the COD analysis. No heavy chain included here in this study but will be put forth elsewhere in the future. Only light chain variable one that is Fab-light-variable one is taken here for study. There are two light chains, kappa and lambda are there separately. The focus is on kappa one here in our study. Specifically human IG kappa variable is analyzed here. 


\section{Methods and Materials}

Both sequence and structure ( $2 \mathrm{KQN}$ ) of human immunoglobulin light chain variable (figure 1) is taken from NCBI database. It is a solution structure containing 108 amino acids. It is supposedly 110 amino acids.

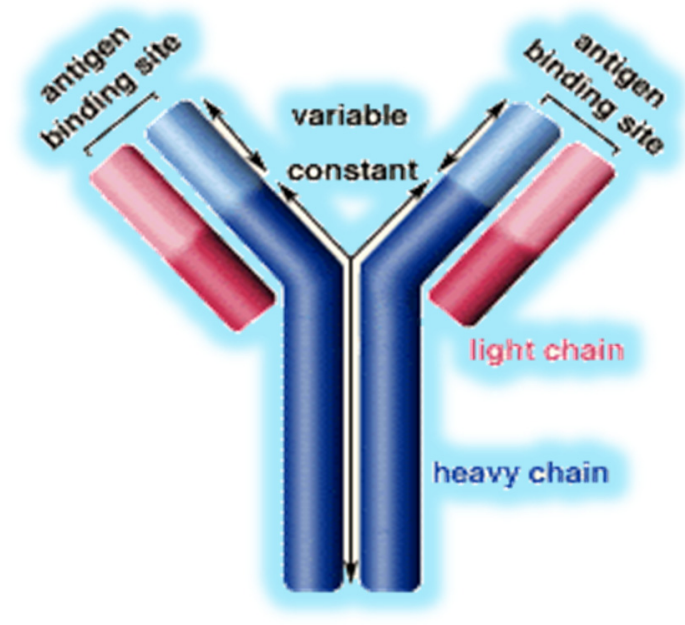

Figure 1. Immunoglobulin variable

Sequence is analyzed using COD program written in PERL script. Average values for atomic length of 70, 105, $140,175,210$ and 245 are taken here. Overall average value is plotted against amino acid as shown in the figure 2. And the other structure analysis is carried out using our home made program, GAD written again in PERL script. The diameter taken as usual D16. It is the mode of interaction taking place while in interacting with another one. So is the $16 \AA$ chosen here for further into all calculations including domain formation and active one leading to binding of other one during intermolecular interaction. The obtained internal COD values are plotted against amino acids as in the figure 3 .

\section{Results and Discussion}

The internal one obtained from sequence analysis is shown in the figure 2. Estimation reveal here that carbon mode of biding due to nonexistence of domain one is clearly revealed here as from 29-35. Evidence shown here are remarkable one available from sequence alone. Neither input nor analysis are difficult to do calculations. Interestingly it is in the indicative that the intervening interaction taken place because of this non domain region obtainable from carbon mode analysis here. Also available is the undomain region, 63-99 which is carbon rich but supposedly buried one. Not available for interaction outside. Only the undomain region, 29-35, exposed outside is available for interaction with incoming molecules. The neighboring domains 18-28 and 36-46 of course in the formation of altered domain during interaction with other one. Alteration favors binding during interaction. It is a remarkable one during evolution in the biological system under study here in antigen antibody interaction. So kappa light value chain variable contain active site at 29-35 which identifiable from COD analysis here. Interestingly the intervening incoming molecule is supposedly eliminate this non exiting carbon that has no domain one. It is cable of capturing the incoming molecule to do some local adjustment the favor binding and all. In this process of interaction only the internal one are altered during interaction. Additional one coming from van der Waals or electrostatics are merely support this phenomena of binding whereas carbon dominates the situation of biding and all. Eliminating this carbon high portion in the active one may be part of the development to neutralize the incoming one not to interfere for study purpose. Overall development in the sequence to full cooperation during interaction is of course be the focus of this work in the future to come. Very much code the intervening one to fulfil the requirement of interaction will be given thrust during the process of development and all. Very much interested in this phenomena of change for the futuristic application of disease free world of course be the focal point in the years to come. Interesting phenomena of carbon role in intervening molecular interaction is to be given boost in the future application and all [17-21]. Energetic lab with dedicated infrastructure facility may of course be the important in this new developmental activity. Very many people are working with valuable knowledge in this direction of work. Given the situation with valuable thought of course be need of this hour. 


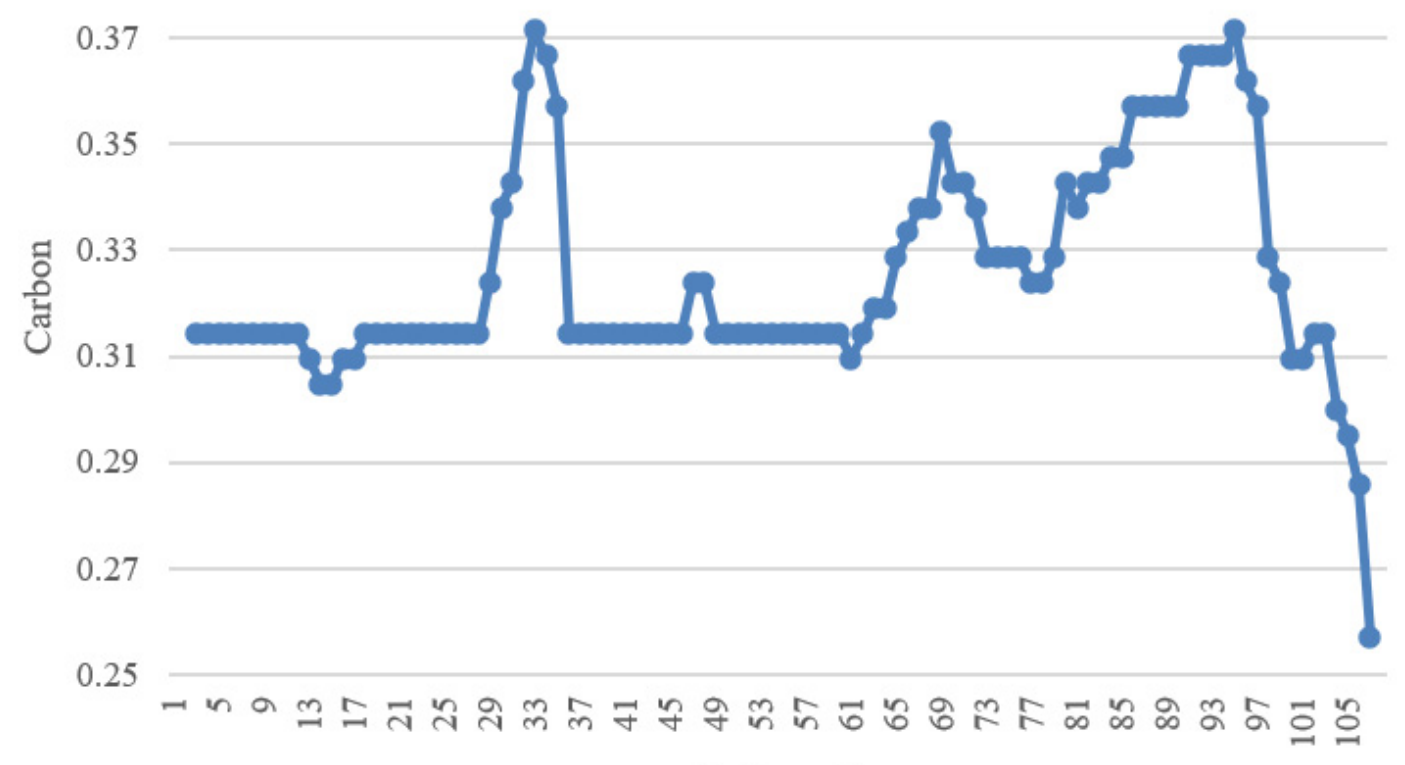

Aminoacid

Figure 2. Average value for different length of atoms obtained from COD one is plotted here against amino acids. Also available the interactive region of interest and domains. The interactive intervening portion of interaction is $29-35$

1-18 and 36-61 are considered to be inactive domain region which has carbon value of $31.45 \%$. Domains are considered as non-interactive portions available during moving around in the system of biological conditions. Alternative to this is the electrophilic regions but again favors this domain value of carbon role. Additional role played by this intervening domain of interaction may be part of the development to careful design and all. Next to this silencing addition of active participant for effective role will be given thrust in the future to come. Addition of active role in improving the binding may also be important for clear decisive and no further improvement over the development may be focus of this study in improvement point of view. Viewing the incoming molecule according to the carbon value will be given important in the process of development. In the vicinity of interaction the incoming molecule sees the internal one to get altered to meaningful adaptation that favors cohesiveness in the internal one of the molecular one. Decisive role played by sequence-structure and all already available with natural phenomena of interaction during evolution that in the database creation. Available are various interactive role played by intervening molecule in the database. Appropriate mating according to the nature and carbon role will be given importance in the system of development. Interestingly the intervening interactive molecule of interaction are almost all available with PDB one. May be searched with carbon threshold for fruitful application and all. Eliminating the bad one and adding active one are to be improved from carbon role point of view. Working with carbon role and adjusting parameter for effective binding are focus of this kind of work planned in the future. Eliminating the bad one where disease solving take place is the most important here in our lab to enrich during evolution in living being into next generation in the context of carbon role from here now onwards. Additional course of interaction may not be sufficient to improve the system of interaction in the years to come and adding new dimension to this carbon role during evolution are considered to be important to give further momentum in the evolution that and all going to be existence once for all. 


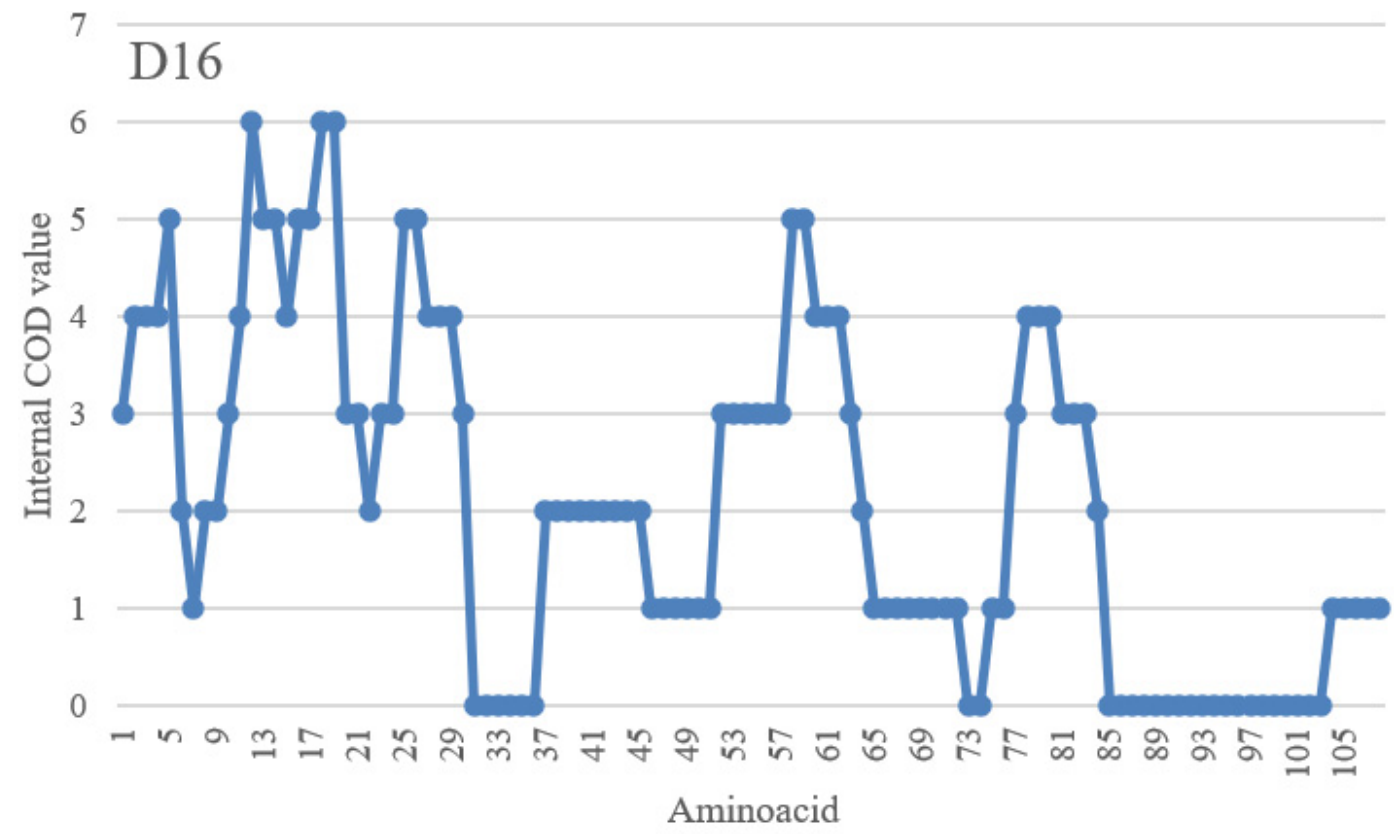

Figure 3. Interaction site obtainable from GAD one is identified as shown here again at 29-35 as there are no elements of domain formed which is responsible for binding of intervening one. Other higher valued one are domains. Otherwise the long zero valued one available are carbon rich site supposedly buried one in the 3D structure

The COD program uses sequence to predict the domain and undomain region the protein molecule of interest. In this context, it is important to note that the available information from sequence one are matched with structure one. One might want to verify this phenomena of internal COD identified from sequence with structural part. It is done here for sample one. Interesting to note that the structural information obtainable from GAD of carbon based calculation produces exact outcome domain values. Available internal COD value are plotted against amino acid position as shown in figure 3. Actually available information are purely from structure one. One might want to correlate with is value of carbon role in the structure which is lineated from sequence again which is amazing. Both are in agreement with each other. Meaning that one might want analyze the source of information from sequence itself rather than going three dimensional structure obtainable from X-Ray or NMR. That is simple sequence analysis can reveal the information of active role played by proteins. Based on this comparison here in antibody antigen interaction [22], it is remarkably matching with sequence one obtained. Again 29-35 identified as active region and other domain regions identified accurately. Also in the plot is seen here the carbon rich region which again comparable but may be some extent non lineated. Over it is all working with prediction.

\section{Conclusion}

One of the most challenging phenomena of binding during antigen antibody interaction is revealed here in terms of carbon value. Adjusting parameter like amino acid sequence is of importance in the years to come. Hoping that the working condition of existing COD and GAD programs be suitable for prediction or development of active site for better futuristic world order in terms of existence of living being and further development activity.

\section{References}

[1] Indupriya R, Meenal R, Kavitha V, and Rajasekaran E, Drug-protein interaction validates the internal COD formed due to cohesive force: Test of bond length variation in amino acids involved, Int J Mol Biol Open Access, 2019; 4: 113-17.

[2] Indupriya R, Meenal R, and Rajasekaran E, Existence of carbon domain alters bond orders in protein, Int $J$ Inno Eng Tech, 2019; 13: 128-32.

[3] Rajasekaran E, Domains based in carbon dictate here the possible arrangement of all chemistry for biology, Int. J. Mol Biol-Open Access, 2018; 3: 240-43.

[4] Rajasekaran E, Meenal R, and Indupriya R Study on aquaporin proves to be the carbon in protein-protein 
interface playing in tetramerisation, High Tech Lett, 2020; 26(5), 292-98.

[5] Rajasekaran E, Meenal R, and Indupriya R Carbon role in the form of action of intelligence in the living being, J Study Res, 2020; 12: 66-72.

[6] Rajasekaran E, Meenal R, Michael PA, and Indupriya R Existence of nano level force in protein plays applications of maximum untold understanding of life form, Int J Eng Adv Tech, 2019; 9: 3722-26.

[7] Rajasekaran E, Meenal R, Indupriya R, Prabakaran R, Boobalan S, Jayato N, Sivakumar K, Kalaivani T, Rathika GM, Saranya K, and Brindha G Existence of cohesive force explains all phenomena that are in material which holds strong bond of all forces of attraction: A case study with carbon material, AIP Conference Proceedings, 2019; 2087, 020015.

[8] Rajasekaran E, Indupriya R, and Meenal R, Domain formation in regions of protein probe interaction, Int $J$ Mol Biol Open Access, 2019; 4: 167-69.

[9] Rajasekaran E, Vinobha CS, Vijayasarathy M, Senthil R and Sankarganesh P, The nature of proteins, Int asso comput sci and info tech, (IACSIT-SC), Singapore, 2009; 464-65.

[10] Rajasekaran E, Scale for nature of hydrophobic interactions in proteins, J Proteomics and Bioinform, 2013; $6(7), 31$.

[11] Rajasekaran E, CARd: Carbon distribution analysis program for protein sequences, Bioinformation, 2012; 8(11), 508-12.

[12] Rajasekaran E, Akila K, Vijayasarathy M, Vinobha CS, Senthil R and Anandagopu P, CARd-3D: Carbon distribution in 3D structure program for globular proteins, Bioinformation, 2014; 10(3), 138-43.

[13] Rajasekaran E, Kavitha V, Ganeshbabu P, Prabakaran R, Meenal R, and Indupriya R, Nature of amino acid sequence instruct carbon value to be adopted in protein 3D structure. IEEE Access, 2019; 1054-60.

[14] Rajasekaran E and Indupriya R, Who power sickle cell disease: Carbon domain analysis tells all because of design in protein 3D arbitrary internal carbon domain (COD) arrangement, Int J Mol Biol Open Access, 2019; 4: $85-88$.

[15] Rajasekaran E, John, SN and Vennila J, Carbon distribution in protein local structure direct superoxide dismutase to disease way, J Proteins and Proteomics, 2012; 3(2), 99-104.

[16] Rajasekaran E, Akila K and Vijayasarathy M, Allotment of carbon is responsible for disorders in proteins, Bioinformation, 2011; 6(8), 291-92.

[17] Amri E, Mamboya AF, Nsimama PD and Rajasekaran E, Role of carbon in crystal structures of wild-type and mutated form of dihydrofolate reductase-thymidylate synthase of P. falciparum, Int J App Bio and Pharma Tech, 2012; 3(3), 1-6.

[18] John SN, Anisha J, Kaarthik AN, Vennila, J and Rajasekaran E, Carbon and protein's half-life, Int J Bioinfo, 2011; 4(2), 23-24.

[19] Nsimama PD, Mamboya AF, Amri E and Rajasekaran E, Correlation between the mutated color tunings and carbon distributions in luciferase bioluminescence, J Comput Intelli in Bioinfo, 2012; 5(2), 105-12.

[20] Rajasekaran E, Jency S and Panneerselvam K, Carbon profile of commercially important sericin protein of silkworm, Bombyx mori, J Adv Bioinfo Appln and Res, 2011; 2(3), 173-76.

[21] Rajasekaran E, Meenal R and Indupriya R, Paradigms in computer vision: Biology based carbon domain postulates nano electronic devices for generation next, In: Hemanth D., Smys S. (eds) Computational vision and bio inspired computing. Lecture notes in computational vision and biomechanics, 2019;28. Springer, Cham.

[22] Mitchell LS, and Colwell LJ, Comparative analysis of nanobody sequence and structure data, Proteins: Structure, function and bioinformatics, 2018; 25497.

\section{Copyrights}

Copyright for this article is retained by the author(s), with first publication rights granted to the journal.

This is an open-access article distributed under the terms and conditions of the Creative Commons Attribution license (http://creativecommons.org/licenses/by/4.0/). 